\title{
Caracterização cromossômica de Anthurium gaudichaudianum (Araceae), uma espécie nativa do Brasil ${ }^{1}$
}

\author{
MARIA GORETI SENNA CORRÊA², JUDITH VIÉGAS ${ }^{2,5}$, \\ MARCUS ALBERTO NADRUZ COELHO ${ }^{3}$, LAUÍS BRISOLARA CORRÊA ${ }^{2} \mathrm{e}$ \\ CARLOS ROGÉRIO MAUCH ${ }^{4}$
}

(recebido: 26 de agosto de 2009; aceito: 26 de agosto de 2010)

\begin{abstract}
Chromosomal characterization of Anthurium gaudichaudianum (Araceae), a native Brazilian species). The karyotype of Anthurium gaudichaudianum Kunth, a species belonging to the Araceae family, is described for the first time. The species is native to Brazil, occurring from the state of Rio Grande do Sul to the state of São Paulo, and the specimens examined were collected from Brazilian Atlantic Forest, in the woods of the municipalities of Torres and Peruíbe, located in the states of Rio Grande do Sul, and São Paulo, Brazil, respectively. Anthurium gaudichaudianum has $2 \mathrm{n}=2 \mathrm{x}=30$ and its karyotype is formed by three pairs of metacentric chromosomes, nine pairs of submetacentric chromosomes and three pairs of acrocentric chromosomes, one of them with satellites $(6 \mathrm{~m}+18 \mathrm{sm}+6 \mathrm{a})$. The size of the chromosomes vary from nearly 6.0 to $2.8 \mu \mathrm{m}$. The complement of 30 chromosomes for A. gaudichaudianum found in our study is in accordance with the findings of $2 \mathrm{n}$ number for most of species in this large genus.
\end{abstract}

Key words - anthuriums, Atlantic Forest, cytogenetics, karyotype, Urospadix section

RESUMO - (Caracterização cromossômica de Anthurium gaudichaudianum (Araceae), uma espécie nativa do Brasil). O cariótipo de Anthurium gaudichaudianum Kunth, uma espécie pertencente à família Araceae, é descrito pela primeira vez. Esta espécie, originária do Brasil, ocorre desde o Estado do Rio Grande do Sul até o de São Paulo e os exemplares analisados foram coletados na Floresta Atlântica brasileira, nas matas nativas dos Municípios de Torres e Peruíbe, localizados respectivamente nos estados do Rio Grande do Sul e de São Paulo. Anthurium gaudichaudianum tem $2 \mathrm{n}=2 \mathrm{x}=30$ e seu cariótipo é formado por três pares de cromossomos metacêntricos, nove pares de cromossomos submetacêntricos e três pares de cromossomos acrocêntricos, um dos quais com satélites $(6 \mathrm{~m}+18 \mathrm{sm}+6 \mathrm{a})$. O tamanho dos cromossomos varia de 6,0 a $2,8 \mu \mathrm{m}$ aproximadamente. $\mathrm{O}$ número $2 \mathrm{n}$ descrito para $A$. gaudichaudianum está de acordo com o complemento cromossômico somático encontrado para a maioria das espécies desse extenso gênero.

Palavras-chave - antúrios, cariótipo, citogenética, Mata Atlântica, seção Urospadix

\section{Introdução}

A família Araceae se divide, atualmente, em 10 subfamílias, possuindo 11 gêneros, incluindo os da família Lemnaceae (Bogner \& Hesse 2003, Gonçalves \& Salviani 2005). Possui cerca de 4.000 espécies distribuídas pela América Tropical e do Norte, África

1. Parte da tese de doutorado da primeira autora, Programa de Pós-Graduação em Agronomia, da Universidade Federal de Pelotas, RS, Brasil.

2. Universidade Federal de Pelotas, Instituto de Biologia, Departamento de Zoologia e Genética, Laboratório de Biologia Celular, Caixa Postal 354, 96010-900 Pelotas, RS, Brasil.

3. Jardim Botânico do Rio de Janeiro, Instituto de Pesquisas, Rua Jardim Botânico, 1008, 22460-070 Rio de Janeiro, RJ, Brasil.

4. Universidade Federal de Pelotas, Faculdade de Agronomia Eliseu Maciel, Departamento de Fitotecnia, Caixa Postal 354, 96010-900 Pelotas, RS, Brasil.

5. Autor para correspondência: juviegas@terra.com.br
Tropical Continental e do Sul, Eurásia Temperada, Arquipélago Malaio, Madagascar e Seychelles. Dessas, 1.100 espécies pertencentes ao gênero Anthurium Schott, considerado o maior desta família, espalham-se por uma área constituída essencialmente pela América Tropical, incluindo países como Argentina, Uruguai e Índias Ocidentais (Mayo et al. 1997). De acordo com Coelho (2007), até 2007 foram descritas 105 espécies desse gênero neotropical no Brasil.

A espécie epifítica Anthurium gaudichaudianum Kunth se localiza no Estado de São Paulo e Região Sul do Brasil, em matas de encosta baixa, alta e campos rupestres. Pode ser reconhecida pela lâmina foliar linearlanceolada a lanceolada com ápice agudo a levemente rostrado e base aguda a obtusa, igualmente estreitada em ambos os lados a levemente mais estreitada para o ápice, com nervuras secundárias obscuras em ambas as faces. Possui longos catafilos inteiros e profilos 
acastanhados a cor de palha, inteiros no ápice, inteiros a decompostos e caducos para a base do caule. $\mathrm{O}$ pedúnculo é esverdeado-vináceo a vináceo. A espata se apresenta ereta a sub-perpendicular em relação à espádice, esverdeada a avermelhado-vinácea, com margens formando ângulo agudo com o pedúnculo. A espádice é vinácea a amarronzada, cilíndrica, estipitada e os frutos são bagas amareladas (Coelho et al. 2004). Essa espécie pertence à seção Urospadix e à subseção Obscureviridia (Keating 2002).

A seção Urospadix Engler agrupa número superior a 54 espécies de Anthurium, concentradas no Leste brasileiro, sendo que mais de $60 \%$ delas ocorrem no Sudeste. A seção é considerada taxonomicamente complexa, com muitas espécies pouco conhecidas e novas para a ciência, adicionando ainda o insuficiente conhecimento das localidades de ocorrência e as escassas coletas nessas áreas. A dificuldade em conseguir posicionar taxonomicamente algumas espécies do gênero Anthurium, como o "complexo harrisii", deve-se principalmente à plasticidade das mesmas que possuem diferenças morfológicas vegetativas e reprodutivas sutis, dando margem a uma taxonomia confusa dentro do grupo (Coelho et al. 2004). Devido à escassez de dados morfológicos para a delimitação taxonômica, fazem-se necessários estudos mais detalhados e confiáveis. Estudos de citotaxonomia, tanto de espécies de difícil determinação taxonômica como para aquelas de taxonomia mais clara, como Anthurium gaudichaudianum, contribuem para o estabelecimento do padrão cromossômico das espécies e das seções.

Okada \& Hotta (1987) e Petersen $(1989,1993)$, ao revisarem a bibliografia sobre o número cromossômico das espécies da família Araceae, verificaram que os números gaméticos relatados variavam de $\mathrm{n}=8$ a $\mathrm{n}=56$ e os somáticos de $2 \mathrm{n}=10$ a $2 \mathrm{n}=168$. Estudos evolutivos sobre a família Araceae e, em especial, sobre o gênero Anthurium, postulam que essa espécie possa ser um anfidiploide, com um número cromossômico básico secundário $\mathrm{x}=15$, o qual foi encontrado na maioria das contagens e que se originou através de hibridações e duplicações, a partir de espécies com números básicos $\mathrm{x}=7$ e $\mathrm{x}=8$ (Sheffer \& Croat 1983, Petersen 1989, Grayum 1990, Cotias-de-Oliveira et al. 1999). Já, Bogner \& Petersen (2007) propoem os números básicos $\mathrm{x}=10,12,14$ e 15 para o gênero Anthurium, em consequência das espécies diploides, tetraploides e hexaploides encontradas. Mayo et al. (1997) ressaltaram o fato de que a maioria das espécies do gênero Anthurium possui número somático diploide de 30 cromossomos. O complemento cromossômico
$2 \mathrm{n}=2 \mathrm{x}=30$ caracteriza a seção Urospadix (Sheffer $\&$ Croat 1983), que possivelmente deve ser uma das seções mais antigas do gênero Anthurium, devido à sua concentração no Leste do Brasil, desde a Bahia até o Rio Grande do Sul (Croat \& Sheffer 1983).

O presente trabalho objetivou, portanto, determinar o número e a morfologia dos cromossomos somáticos de Anthurium gaudichaudianum coletados na Mata Atlântica, no Sudeste e Sul do Brasil.

\section{Material e métodos}

Os exemplares de Anthurium gaudichaudianum estudados foram coletados na Mata Atlântica, nos Municípios de Torres, Estado do Rio Grande do Sul (Voucher PEL 14536) e de Peruíbe, Estado de São Paulo (Voucher Nadruz 1477, RB/JBRJ), Brasil. Mantiveram-se mudas em frascos de vidro com solução nutritiva $0,03 \%$ (Viégas et al. 2007) ou em solo vegetal, das quais foram utilizadas pontas de raiz jovens, com cerca de um a dois centímetros de comprimento.

As pontas de raiz foram pré-tratadas com 8-HQ $0,002 \mathrm{M}$ a $4 \pm 2{ }^{\circ} \mathrm{C}$ por 24 horas, com ou sem a imersão prévia neste agente antimitótico, por uma a duas horas, em temperatura ambiente. O material foi fixado em 6:3:1 (álcool etílico: clorofórmio: ácido acético), por duas a 24 horas, e estocado em álcool $70 \%$, a $4 \pm 2{ }^{\circ} \mathrm{C}$, até sua utilização. Para a preparação das lâminas, o material foi hidrolisado em $\mathrm{HCl}$ $5 \mathrm{~N}$ por 45 minutos, à temperatura ambiente, seguido ou não de 10 minutos em solução enzimática (celulase $2 \%$ e pectinase 20\%, em tampão fosfato citrato, $\mathrm{pH} 4,8$ ), em câmara úmida, a $37 \pm 1{ }^{\circ} \mathrm{C}$. O meristema radicular foi esmagado em ácido acético $45 \%$ e todas as lâminas foram tornadas permanentes por congelamento em nitrogênio líquido. Após secagem ao ar e coloração em solução de Giemsa $2 \%$, a montagem do preparado foi feita com Entellan.

As melhores placas metafásicas foram fotografadas com filme colorido Kodak de sensibilidade de ISO 100 e as fotos foram utilizadas para a análise morfométrica dos cromossomos. A fórmula cariotípica foi obtida através da média da medida dos cromossomos de 20 placas metafásicas, baseada no índice centromérico (ic): ic $=\mathrm{c} \cdot 100 / \mathrm{c}+1$, onde $\mathrm{c}=$ braço curto e $1=$ braço longo. Os cromossomos foram classificados de acordo com Guerra (1986) em metacêntricos (ic $=50,0$ a 40,1), submetacêntricos (ic $=40,0$ a 25,1), acrocêntricos (ic $=25,0$ a 0,01$)$ e telocêntricos $(i c=0)$.

\section{Resultados e discussão}

O complemento cromossômico encontrado para Anthurium gaudichaudianum é diploide $2 \mathrm{n}=2 \mathrm{x}=30$ (figuras 1 e 2) e se apresenta em acordo com o da maioria das espécies do gênero. Sheffer \& Croat (1983) analisaram e/ou revisaram um total de 137 espécies de Anthurium, tendo encontrado uma faixa de variação de 
$2 n=24$ a $2 n=90$, dentro da qual: a) 74,4\% das espécies tinham o complemento diploide $2 \mathrm{n}=2 \mathrm{x}=30$; b) $9,5 \%$ apresentaram contagens de $2 n=2 x=30$ e de $2 n=2 x \neq 30$, nas quais provavelmente os números diferentes de 30 se devem à presença de cromossomos $\mathrm{B}$, fragmentos ou a contagens duvidosas em função da adesividade cromossômica; c) 4,4\% das espécies eram tetraploides $2 \mathrm{n}=4 \mathrm{x}=60 ;$ d) $4,4 \%$ apresentaram exemplares com $2 n=2 x=30$ e $2 n=4 x=60$ na mesma espécie; e) $0,7 \%$ das espécies eram hexaploides $2 n=6 x=90$ e f) $6,6 \%$ apresentaram vários números cromossômicos, variando de $2 \mathrm{n}=24$ a $2 \mathrm{n}=34$, com uma única espécie com contagem cromossômica superior a 90, A. lucidum Kunth, com $2 \mathrm{n}=c a$. 124. Na verdade, pode-se considerar a frequência de espécies diploides do gênero Anthurium, como sendo $83,9 \%$, pois o grupo (b) pode ser reunido ao (a).

Baseados no número diploide dos cromossomos somáticos, apresentado pela maioria das espécies de antúrios, Mayo et al. (1997) consideraram este gênero citologicamente uniforme. O estudo citotaxonômico,
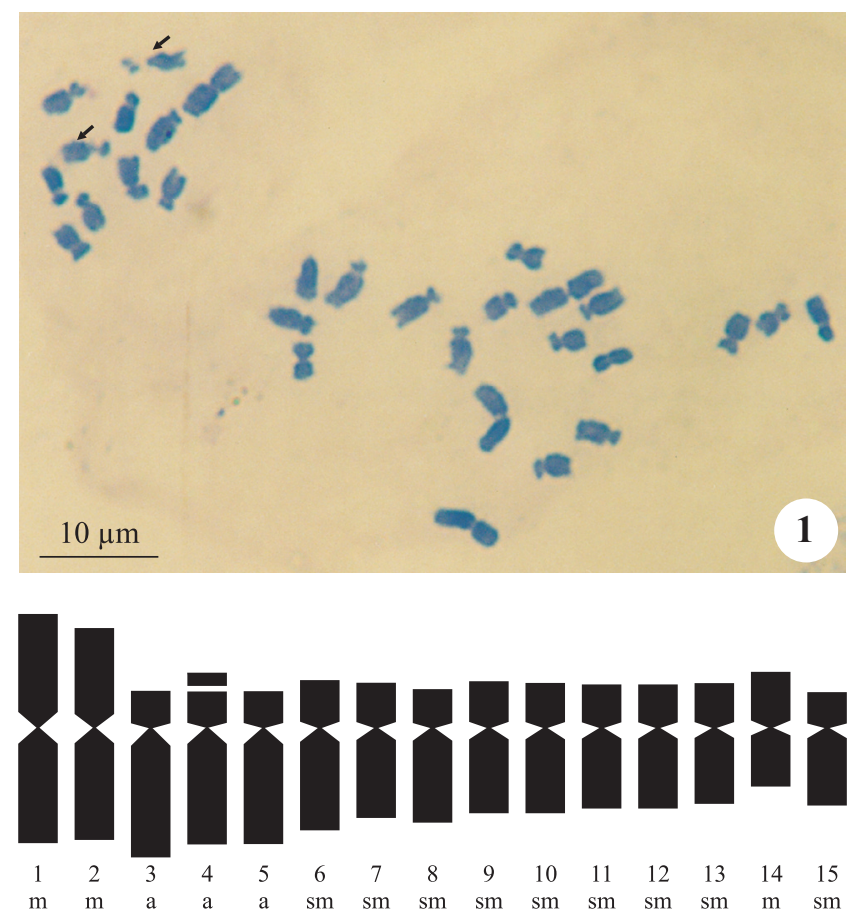

Figuras 1 e 2. 1. Cromossomos somáticos de Anthurium gaudichaudianum, $2 \mathrm{n}=2 \mathrm{x}=30$ ( $\operatorname{setas}=$ cromossomos satelitados). 2. Idiograma de Anthurium gaudichaudianum $(\mathrm{m}=$ metacêntrico; $\mathrm{sm}=$ submetacêntrico; $\mathrm{a}=$ acrocêntrico $)$.

Figures 1 and 2. 1. Anthurium gaudichaudianum somatic chromosomes $2 \mathrm{n}=2 \mathrm{x}=30$ (arrows $=$ satellite chromosomes). 2. Anthurium gaudichaudianum idiogram $(\mathrm{m}=$ metacentric; $\mathrm{sm}=$ submetacentric; $\mathrm{a}=$ acrocentric) . realizado por Dhanavel \& Subramanian (2004), mostrou complemento cromossômico $2 \mathrm{n}=30$ e $2 \mathrm{n}=16$ para as espécies $A$. cubense Engl. e A. polyrrhizum K. Koch \& Augustin (sinonímia de A. rubrinervium (Link) G. Don), respectivamente. Mohamed et al. (2006) confirmaram o número diplóide $2 \mathrm{n}=2 \mathrm{x}=30$ de $A$. andraeanum Linden e de $A$. hookeri Kunth, ao estudarem a variabilidade cromossômica de alguns gêneros da família Araceae. Viégas et al. (2006) ressaltaram que, das espécies de Anthurium analisadas posteriormente ao trabalho de Sheffer \& Croat (1983), cerca de 74,6\% também foram relatadas como diploides.

A maioria das espécies de antúrios, analisadas citogeneticamente, teve o seu número cromossômico somático relatado nos artigos de Marutani et al. (1988, 1993), que apresentaram os cariótipos de algumas espécies das seções Calomystrium Schott emend Engl.; Polyneurium Engl.; Semaeophyllium Schott; Belolonchium Schott (tabela 1). Conforme a tabela 1, verifica-se que os dois pares de cromossomos grandes apresentam comprimentos médios de 6,0 e 5,2 $\mu \mathrm{m}$, sendo que os demais cromossomos são de tamanho médio a pequeno e têm, emmédia, cerca de 4,1 a 2,8 $\mu \mathrm{m}$. Anthurium gaudichaudianum possui, portanto, cromossomos de tamanho similar aos de algumas espécies do gênero, tais como $A$. warocqueanum T. Moore, que possui dois pares grandes, com aproximadamente $6,0 \mu \mathrm{m}$, e os pares médios a pequenos variam de 4,0 a 2,8 $\mu \mathrm{m}$ (Kaneko \& Kamemoto 1979). Os cromossomos também se assemelham aos das espécies brasileiras $A$. affine Schott, A. longipes N.E. Br, A. pentaphyllum (Aubl.) G. Don var. pentaphyllum e $A$. bellum Schott, que apresentaram tamanho entre 6,7 a 2,5 $\mu \mathrm{m}$ (Cotias-de-Oliveira et al. 1995). Os dados morfométricos dos cromossomos de $A$. gaudichaudiaum não coincidem com os de $A$. scherzerianum Schott, que ficam numa faixa de 3,8 a $1,6 \mu \mathrm{m}$ (Subramanian \& Munian 1988) ou com os de A. clidemioides Standl. e A. gymnopus Griseb., com 4 a $2 \mu \mathrm{m}$ e 4 a $3 \mu \mathrm{m}$, respectivamente (Petersen 1989).

Os cromossomos grandes de $A$. gaudichaudianum são metacêntricos. Na classe de cromossomos de tamanho médio, os acrocêntricos são facilmente visualizados por serem os de maior comprimento. Os demais cromossomos são submetacêntricos e apresentam uma diminuição gradual de tamanho. O par cromossômico 14 se destaca dos demais cromossomos de tamanho pequeno por ser metacêntrico (tabela 1). O cariótipo é constituído, portanto, de dois pares de cromossomos metacêntricos grandes, um par de cromossomos metacêntricos pequenos, nove pares de cromossomos submetacêntricos e três pares de cromossomos acrocêntricos médios, 
Tabela 1. Morfologia dos cromossomos somáticos de células de ponta de raiz de Anthurium gaudichaudianum $(\mathrm{m}=$ metacêntrico; sm = submetacêntrico; $\mathrm{a}=$ acrocêntrico) .

Table 1. Morphology of root cells somatic chromosomes of Anthurium gaudichaudianum $(\mathrm{m}=$ metacentric; sm = submetacentric; $\mathrm{a}=$ acrocentric).

\begin{tabular}{|c|c|c|c|c|c|}
\hline \multirow{2}{*}{$\begin{array}{l}\text { Cromossomo } \\
\text { número }\end{array}$} & \multicolumn{3}{|c|}{$\begin{array}{l}\text { Medidas sobre ampliação fotográfica } \\
\qquad(\mathrm{mm})\end{array}$} & \multirow{2}{*}{$\begin{array}{l}\text { Morfologia } \\
\text { cromossômica }\end{array}$} & \multirow{2}{*}{$\begin{array}{l}\text { Medida em } \\
\mu \mathrm{m}\end{array}$} \\
\hline & $\begin{array}{l}\text { Braço curto } \\
\text { (c) }\end{array}$ & $\begin{array}{l}\text { Braço longo } \\
\text { (l) }\end{array}$ & $\begin{array}{l}\text { Índice centromérico } \\
\quad \mathrm{ic}=\mathrm{c} \cdot 100 / \mathrm{c}+1\end{array}$ & & \\
\hline 1 & 6,00 & 6,91 & 46,47 & $\mathrm{~m}$ & 6,0 \\
\hline 2 & 5,25 & 5,92 & 47,00 & $\mathrm{~m}$ & 5,2 \\
\hline 3 & 1,91 & 6,85 & 21,81 & $\mathrm{a}$ & 4,1 \\
\hline 4 & 1,84 & 6,16 & 23,00 & $\mathrm{a}$ & 3,7 \\
\hline 5 & 1,87 & 6,12 & 23,40 & $\mathrm{a}$ & 3,7 \\
\hline 6 & 2,58 & 5,41 & 32.29 & $\mathrm{sm}$ & 3,7 \\
\hline 7 & 2,34 & 4,75 & 33.00 & $\mathrm{sm}$ & 3,3 \\
\hline 8 & 2,00 & 5,00 & 28.57 & $\mathrm{sm}$ & 3,3 \\
\hline 9 & 2,42 & 4,50 & 34.97 & $\mathrm{sm}$ & 3,3 \\
\hline 10 & 2,33 & 4,50 & 34.15 & $\mathrm{sm}$ & 3,2 \\
\hline 11 & 2,25 & 4,25 & 34.62 & $\mathrm{sm}$ & 3,0 \\
\hline 12 & 2,25 & 4,25 & 34.62 & $\mathrm{sm}$ & 3,0 \\
\hline 13 & 2,32 & 4,00 & 36.70 & $\mathrm{sm}$ & 2,9 \\
\hline 14 & 2.92 & 3,08 & 48.67 & $\mathrm{~m}$ & 2,8 \\
\hline 15 & 1,84 & 4,09 & 31.03 & $\mathrm{sm}$ & 2,8 \\
\hline
\end{tabular}

sendo um deles com satélite (figuras 1 e 2). A fórmula cariotípica é $6 \mathrm{~m}+18 \mathrm{sm}+6 \mathrm{a}$.

Conforme a tabela 2 , o cariótipo de $A$. gaudichaudianum se assemelha ao de A. amnicola pela presença de quatro cromossomos metacêntricos grandes e seis acrocêntricos médios, mas o primeiro possui dois submetacêntricos médios. Além disso, os 20 cromossomos pequenos de $A$. amnicola são citados como sendo metacêntricos e acrocêntricos (Marutani et al. 1988). Também como A. gaudichaudianum, 10 espécies de Anthurium, analisadas por Marutani et al. (1993), apresentaram quatro cromossomos grandes metacêntricos e/ou submetacêntricos, mas apenas dois acrocêntricos médios. Neste mesmo artigo, verifica-se que $A$. nymphaeifolium e A. ochranthum fugiram desse padrão cariotípico: A. nymphaeifolium apresentou dois cromossomos grandes metacêntricos e dois grandes acrocêntricos, com ausência dos dois acrocêntricos médios, enquanto que $A$. ochranthum possuía dois cromossomos médios metacêntricos. Os autores propuseram que a variação encontrada nestes dois últimos cariótipos foi devida a rearranjos cromossômicos ocorridos no cariótipo típico $(4 \mathrm{~m} / \mathrm{sm}+2 \mathrm{a})$. Verifica-se, também, na tabela 2 , que excetuando $A$. gaudichaudianum, cujos cromossomos pequenos tiveram sua morfologia bem estabelecida, e A. amnicola, onde estes foram descritos como metacêntricos e acrocêntricos (Marutani et al. 1988), para as demais espécies estudadas houve apenas a citação de número de cromossomos pequenos (Marutani et al. 1993), demonstrando a dificuldade em determinar a morfologia dos mesmos quando apresentam dimensões diminutas.

Ramalho (1995) descreveu espécies ocorrentes em Pernambuco, Brasil, entre elas A. gracile (Rudge) Schott $(2 \mathrm{n}=2 \mathrm{x}=40)$ e A. olfersianum Kunth (= cf. parasiticum $)(2 \mathrm{n}=2 \mathrm{x}=30)$, ambas com cromossomos metacêntricos, submetacêntricos e acrocêntricos. As demais espécies analisadas por Ramalho (1995) não apresentaram acrocêntricos: A. affine e A. petrophilum K. Krause $(\operatorname{ambos} 2 \mathrm{n}=2 \mathrm{x}=30)$, A. pentaphyllum var. pentaphyllum $(2 \mathrm{n}=4 \mathrm{x}=60)$ e $A$. scandens (Aubl.) Engl. ssp. scandens $(2 \mathrm{n}=2 \mathrm{x}=48)$.

Cotias-de-Oliveira et al. (1999), estudando antúrios endêmicos da Bahia - Brasil, verificaram que A. affine $(2 \mathrm{n}=2 \mathrm{x}=30)$ apresenta oito pares de cromossomos metacêntricos e sete de submetacêntricos e que em $A$. longipes $(2 \mathrm{n}=2 \mathrm{x}=30)$ predominamos submetacêntricos. Estes autores encontraram $2 \mathrm{n}=2 \mathrm{x}=30$ e $2 \mathrm{n}=4 \mathrm{x}=60$ para A. pentaphyllum var. pentaphyllum e $2 \mathrm{n}=6 \mathrm{x}=90$ para A. bellum.

Cromossomos pequenos, com comprimento de aproximadamente 1 a $3 \mu \mathrm{m}$, geralmente ocorrem em 
Tabela 2. Descrição do cariótipo, morfologia e tamanhos cromossômicos de espécies do gênero Anthurium Schott de trabalhos publicados após o de Sheffer \& Croat (1983) (m = metacêntrico; sm = submetacêntrico; a = acrocêntrico; ? = morfologia não descrita; ${ }^{(1),(2)}=\mathrm{n}^{\mathrm{o}}$ de cromossomos B).

Table 2. Karyotype, chromosome morphology, and chromosome length description of Anthurium Schott species in paper published after Sheffer \& Croat (1983) ( $\mathrm{m}=$ metacentric; sm = submetacentric; a = acrocentric; ? = morphology undescribed; (1), (2) $=$ chromosome B number).

\begin{tabular}{|c|c|c|c|c|c|c|}
\hline \multirow{2}{*}{ Autor, ano } & \multicolumn{2}{|c|}{$\begin{array}{c}\text { Gênero } \\
\text { Anthurium Schott }\end{array}$} & \multirow{2}{*}{$\begin{array}{c}\text { Cariótipo } \\
2 \mathrm{n}=2 \mathrm{x}=30\end{array}$} & \multicolumn{3}{|c|}{$\begin{array}{c}\text { Cromossomos } \\
\text { Morfologia eTamanho }\end{array}$} \\
\hline & Seções & Espécies & & Grandes & Médios & Pequenos \\
\hline $\begin{array}{l}\text { Presente } \\
\text { trabalho } \\
(2010)\end{array}$ & Urospadix & $\begin{array}{l}\text { A. gaudichaudianum } \\
\text { Kunth }\end{array}$ & $6 \mathrm{~m}+6 \mathrm{a}+18 \mathrm{sm}^{(1)}$ & $4 \mathrm{~m}$ & $6 a+2 s m$ & $\begin{array}{c}16 \mathrm{sm}+ \\
2 \mathrm{~m}\end{array}$ \\
\hline $\begin{array}{l}\text { Marutani } \\
\text { et al. (1988) }\end{array}$ & Calomystrium & A. amnicola Dressler & $4 m+6 a+20 m / a$ & $4 \mathrm{~m}$ & $6 \mathrm{a}$ & $20 \mathrm{~m} / \mathrm{a}$ \\
\hline \multirow[t]{4}{*}{$\begin{array}{l}\text { Marutani } \\
\text { et al. (1993) }\end{array}$} & Calomystrium & $\begin{array}{l}\text { A. andraeanum Linden } \\
\text { A. formosum Schott } \\
\text { A. kamemotoanum Croat } \\
\text { A. lindeneanum Schott } \\
\text { A. roseospadix Croat } \\
\text { A. cf. sanctifidense Croat } \\
\text { Anthurium sp. } \\
\text { A. nymphaeifolium } \\
\text { K. Kouch \& Bouché }\end{array}$ & $\begin{array}{l}4 \mathrm{~m} / \mathrm{sm}+2 \mathrm{a}+24 ? \\
4 \mathrm{~m} / \mathrm{sm}+2 \mathrm{a}+24 ? \\
4 \mathrm{~m} / \mathrm{sm}+2 \mathrm{a}+24 ? \\
4 \mathrm{~m} / \mathrm{sm}+2 \mathrm{a}+24 ? \\
4 \mathrm{~m} / \mathrm{sm}+2 \mathrm{a}+24 ? \\
4 \mathrm{~m} / \mathrm{sm}+2 \mathrm{a}+24 ? \\
4 \mathrm{~m} / \mathrm{sm}+2 \mathrm{a}+24 ? \\
2 \mathrm{~m}+2 \mathrm{a}+26 ?\end{array}$ & $\begin{array}{l}4 \mathrm{~m} / \mathrm{sm} \\
4 \mathrm{~m} / \mathrm{sm} \\
4 \mathrm{~m} / \mathrm{sm} \\
4 \mathrm{~m} / \mathrm{sm} \\
4 \mathrm{~m} / \mathrm{sm} \\
4 \mathrm{~m} / \mathrm{sm} \\
4 \mathrm{~m} / \mathrm{sm} \\
2 \mathrm{~m}+2 \mathrm{a}\end{array}$ & $\begin{array}{l}2 \mathrm{a} \\
2 \mathrm{a} \\
2 \mathrm{a} \\
2 \mathrm{a} \\
2 \mathrm{a} \\
2 \mathrm{a} \\
2 \mathrm{a} \\
-\end{array}$ & $\begin{array}{l}24 ? \\
24 ? \\
24 ? \\
24 ? \\
24 ? \\
24 ? \\
24 ? \\
26 ?\end{array}$ \\
\hline & Semaeophyllium & $\begin{array}{l}\text { A. subsignatum Schott } \\
\text { A. garagaranum Standl. }\end{array}$ & $\begin{array}{l}4 \mathrm{~m} / \mathrm{sm}+2 \mathrm{a}+24 ? \\
4 \mathrm{~m} / \mathrm{sm}+2 \mathrm{a}+24 ?^{(1)}\end{array}$ & $\begin{array}{l}4 \mathrm{~m} / \mathrm{sm} \\
4 \mathrm{~m} / \mathrm{sm}\end{array}$ & $\begin{array}{l}2 \mathrm{a} \\
2 \mathrm{a}\end{array}$ & $\begin{array}{l}24 ? \\
24 ?\end{array}$ \\
\hline & Polyneurium & $\begin{array}{l}\text { A. caperatum Croat \& } \\
\text { R. Baker }\end{array}$ & $4 \mathrm{~m} / \mathrm{sm}+2 \mathrm{a}+24 ?$ & $4 \mathrm{~m} / \mathrm{sm}$ & $2 \mathrm{a}$ & $24 ?$ \\
\hline & Belolonchium & A. ochranthum K. Koch & $6 \mathrm{~m} / \mathrm{sm}+24 ?^{(2)}$ & $4 \mathrm{~m} / \mathrm{sm}$ & $2 \mathrm{~m}$ & $24 ?$ \\
\hline
\end{tabular}

plantas com posição evolutiva ancestral. Nos gêneros, nas tribos e nas subfamílias, que ocupam posições basais na filogenia da família Araceae, observa-se uma esmagadora ocorrência de espécies com cromossomos pequenos. No entanto, parece que muitos gêneros dessa família, evolutivamente mais recentes, mantiveram os primitivos cromossomos de tamanho pequeno. Em relação à morfologia, cromossomos acrocêntricos e telocêntricos parecem ter sido confinados aos gêneros com cromossomos médios ou grandes. Assim, nas Araceae, de modo geral, os passos evolutivos parecem ter ocorrido no sentido de cromossomos metacêntricos pequenos a cromossomos grandes com centrômeros mais distais (Petersen 1993). Com base em seu cariótipo, A. gaudichaudianum pode ser classificado em uma posição evolutiva intermediária, devido à presença de cromossomos com morfologia acrocêntrica e submetacêntrica e de tamanho grande a pequeno.

Ao lado do interesse puramente científico de uma análise teórica, os dados apresentados no presente trabalho agregam informações importantes aos estudos taxonômicos de cerca de $10 \%$ das espécies atualmente conhecidas do gênero Anthurium, que são as espécies brasileiras. Informações, estas, que serão extremamente úteis não só do ponto de vista taxonômico, mas também do ponto de vista genético-evolutivo, principalmente no que se refere ao estabelecimento do centro de origem das diversas seções.

Agradecimentos - Ao Prof. Dr. J. A. Jarenkow (Departamento de Botânica, IB/UFRGS), pela coleta do material no município de Torres, RS e à Capes, pela bolsa de doutorado.

\section{Referências bibliográficas}

BOGNER, J. \& HESSE, M. 2003. Zamioculcadoideae, a new subfamily of Araceae. CNIP. Checklist das plantas do nordeste. http://umbuzeiro. cnip.org.br/db/pnechk/taxa/321.html (acesso em 20/08/2005). 
BOGNER, J. \& PETERSEN, G. 2007. The chromosome numbers of the Aroid genera. Aroideana 30:82-90.

COELHO, M.A.N. 2007. Araceae do estado de São Paulo. In Livro vermelho das espécies ameaçadas do estado de São Paulo. (M.H.C. Mamede, V.C. Souza, J. Prado, F. Barros, M.G.L. Wanderley \& J.G. Rando, eds.) Instituto de Botânica, São Paulo, p.53-56.

COELHO, M.A.N., WAECHTER, J.L. \& MAYO, S.M. 2004. Taxonomia e biogeografia de Anthurium Schott. (Araceae) seção Urospadix subseção Flavescentiviridia. Tese de doutorado, Universidade Federal do Rio Grande do Sul, Porto Alegre.

COTIAS-DE-OLIVEIRA, A.L.P., BARRETO, E.C., SANTOS, S.B.\& FERNANDES, F.D. 1995. Citogenética de espécies de Anthurium (Araceae). Revista Brasileira de Genética 18:409.

COTIAS-DE-OLIVEIRA, A.L.P., GUEDES, M.L. \& BARRETO, E.C. 1999. Chromosome numbers for Anthurium and Philodendron spp. (Araceae) occurring in Bahia. Genetics and Molecular Biology 22: 237-242.

CROAT, T.B. \& SHEFFER, R.D. 1983. The sectional groupings of Anthurium (Araceae). Aroideana 6:85-123.

DHANAVEL, D. \& SUBRAMANIAN, D. 2004. Cytotaxonomical studies in Indian Araceae. Plant Archives 4:81-88.

GONÇALVES, E.G. \& SALVIANI, E.R. 2005. Two new Andean genera for the tribe Spathicarpeae (Araceae). Willdenowia 35:319-326.

GRAYUM, M.H. 1990. Evolution and phylogeny of the Araceae. Annals of the Missouri Botanical Garden 77:628-697.

GUERRA, M.S. 1986. Reviewing the chromosome nomenclature of Levan et al. Revista Brasileira de Genética 9:741-743.

KANEKO, K. \& KAMEMOTO, H. 1979. Karyotype and B chromosome of Anthurium warocqueanum. The Journal of Heredity 70:271-272.

KEATING, R.C. 2002. Anatomy of the monocotyledons IX. Acoraceae and Araceae. Clarendon Press, Oxford.
MARUTANI, M., WANNAKRAIROJ, S. \& KAMEMOTO, H. 1988. Chromosome studies on Anthurium amnicola and its hybrids. Aroideana 11:9-14.

MARUTANI, M., SHEFFER, R.O. \& KAMEMOTO, H. 1993. Cytological analysis of Anthurium andraeanum (Araceae) its related taxa and their hybrids. American Journal of Botany 80:93-103.

MAYO, S.J., BOGNER, J. \& BOYCE, P.C. 1997. The genera of Araceae. Royal Botanical Garden, Kew.

MOHAMED, T.R., KHALIFA, S.F. \& SALAH EL-DINE, R.M. 2006. Leaf protein electrophoretic profiles and chromosome numbers of some Araceae. International Journal of Agriculture \& Biology 8:231-234.

OKADA, H. \& HOTTA, M. 1987. Chromosome numbers of Araceae. Biological Laboratory of the Kyoto University, Kyoto.

PETERSEN, G. 1989. Cytology and systematics of Araceae. Nordic Journal of Botanic 9:119-166.

PETERSEN, G. 1993. Chromosome numbers of the genera of Araceae. Aroideana 16:37-46.

RAMALHO, F.C. 1995. Taxonomia e número cromossômico de representantes da família Araceae em Pernambuco. Dissertação de mestrado, Universidade Federal Rural Pernambuco, Recife.

SHEFFER, R.D. \& CROAT, T.B. 1983. Chromosome numbers in the genus Anthurium (Araceae). II. American Journal of Botany 70:858-871.

SUBRAMANIAN,D.\&MUNIAN,M. 1988.Cytotaxonomical studies in South Indian Araceae. Cytologia 53:59-66.

VIÉGAS, J., COELHO, M.A.N., CORRÊA, M.G.S. \& CORRÊA, L.B. 2006. Taxonomic and cytogenetic analysis of species of the Anthurium (Araceae) genus native to the Brazilian Atlantic Forest. In Floriculturure, ornamental and plant biotechnology (J.T. Silva, ed.). Global Science Books, Middlesex, v. IV, p.669-677.

VIÉGAS, J., ROCHA, M.T.R., FERREIRA-MOURA, I., ROSA, D.L., SOUZA, J.A., CORRÊA, M.G.S. \& SILVA, J.T. 2007. Anthurium andraeanum (Linden ex André) culture: in vitro and ex vitro. Floriculture and Ornamental Biotechnology 1:61-65. 